\title{
Fiabilidade da Escala de Conhecimento da Diabetes em Portugal
}

\author{
Diabetes Knowledge Test Feasibility in Portugal
}

\author{
Constança AZEVEDO $\mathbb{1}^{1}$, Luiz SANTIAGO ${ }^{2,3}$
}

Acta Med Port 2016 Sep;29(9):499-506 - http://dx.doi.org/10.20344/amp.7517

RESUMO

Introdução: Dada a crescente incidência da diabetes tipo 2 e considerando que parte do seu controlo se deve à atitude do paciente face à doença, torna-se importante munir os profissionais de saúde com ferramentas capazes de determinar as carências educacionais dos utentes, permitindo uma intervenção mais personalizada na correção de hábitos nocivos.

Objetivos: Verificação da fiabilidade da Escala de Conhecimentos da Diabetes em Portugal. Análise e correlação das diferentes variáveis sociodemográficas e patológicas com o número de respostas corretas.

Material e Métodos: Aplicação da escala a uma amostra de conveniência de diabéticos tipo 2 do Centro de Saúde da Covilhã.

Resultados: Na generalidade, as questões obtiveram um valor de alfa de Cronbach $>0,800$. Diabéticos com melhores resultados demonstraram maior controlo, estabelecendo uma relação positiva entre o conhecimento e o controlo da diabetes. Apenas $9,2 \%$ dos diabéticos apresentou bom conhecimento sobre a doença, sendo que a maioria $(65,8 \%)$ demonstrou um conhecimento mediano. Os insulinotratados exibiram os piores resultados ( $77,6 \% \mathrm{com}$ baixo conhecimento). As variáveis 'via de tratamento' e 'complicações' demonstraram ter impacto no desempenho obtido no questionário (valores $p$ iguais a 0,00 e 0,048 , respetivamente). Os diabéticos rurais, os sem complicações e os não insulinotratados revelaram-se os mais cumpridores e conhecedores.

Discussão: Contrariando estudos anteriores, variáveis como a idade e o grau de escolaridade não demonstraram influenciar o conhecimento do diabético. Habitantes do meio rural e diabéticos tratados oralmente obtiveram melhores resultados do que o que havia sido defendido previamente.

Conclusão: Demonstrou-se a fidelidade psicométrica moderada a elevada do teste, assim como uma correlação positiva entre o controlo da diabetes e o desempenho no inquérito. Espera-se que a sua futura aplicação possibilite identificar as razões do descontrolo dos diabéticos, permitindo uma atuação mais individualizada pelos profissionais de saúde.

Palavras-chave: Avaliação Educacional; Conhecimentos, Atitudes e Prática em Saúde; Diabetes Mellitus; Portugal; Psicometria.

\section{ABSTRACT}

Introduction: Given the rising incidence of type 2 diabetes and taking into account that much of its control depends on the patient's attitude towards his illness, loomed the need to equip health professionals with capable tools to determine the educational needs of their patients, allowing a more personalized intervention to correct habits that could be harmful to the diabetics' health.

Objective: Verify the Diabetes Knowledge Test reliability in Portugal. Analysis of socio-demographic and pathologic variables' correlation with the number of correct answers in the test.

Material and Methods: Application of the translated version of Diabetes Knowledge Test to a convenience sample of a primary care center in Covilhã.

Results: In general, the questions obtained a Cronbach's alpha $>0.800$. Diabetics with better results in the survey proved to be more compliant, with a positive relationship between knowledge and diabetes' control. However, only $9.2 \%$ of diabetics have good knowledge about their disease, and the majority (65.8\%) shows an average one. The insulin treated patients show the worst results $(77.6 \%$ with low knowledge). The variables 'treatment route' and 'complications' show statistical impact on the level of knowledge that diabetics have about their pathology ( $p$ values 0.00 and 0.048 , respectively). Inhabitants of rural areas, without complications secondary to diabetes and treated orally, proved to be more compliant and to have better knowledge about the disease.

Discussion: Contrary to previous studies, variables such as age and educational level showed no relation with the performance in the survey. Inhabitants of the rural area and diabetics treated orally obtained better results than what had been previously advocated. Conclusion: It has been proved the moderate to high psychometric reliability of the test, as its value establishing a correlation between diabetes knowledge and control. It is expected that its future application will help identifying the reasons for uncontrolled diabetics, allowing a more individualized intervention by the health professionals.

Keywords: Diabetes Mellitus; Educational Measurement; Health Knowledge, Attitudes, Practice; Portugal; Psychometrics.

\section{INTRODUÇÃO}

De acordo com a Organização Mundial da Saúde, ${ }^{1}$ a diabetes mellitus tipo 2 (DM2), afeta cerca de $90 \%$ da população diabética mundial. Dada a sua crescente incidência, e tendo em consideração que $95 \%$ do seu controlo se deve à atitude do paciente face à sua doença, assomou-se a necessidade de munir os profissionais de saúde de ferramen-

tas capazes de determinar as carências educacionais dos seus utentes ${ }^{2-7}$ Assim, o Michigan Diabetes Research and Training Center desenvolveu a Escala de Conhecimentos da Diabetes (Diabetes Knowledge Test [DKT]) que permite avaliar o conhecimento que o diabético tem sobre as implicações da sua doença a nível alimentar, do estilo de vida

1. Estudante. Faculdade de Ciências da Saúde. Universidade da Beira Interior. Covilhã. Portugal.

2. USF Topázio. Coimbra. Portugal.

3. Universidade da Beira Interior. Covilhã. Portugal.

$\triangle$ Autor correspondente: Constança Azevedo. a26729@fcsaude.ubi.pt

Recebido: 14 de fevereiro de 2016 - Aceite: 26 de abril de 2016 | Copyright $\odot$ Ordem dos Médicos 2016 
e manuseio da terapêutica. ${ }^{2}$ Ao identificar as necessidades educativas dos diabéticos nestas temáticas, o DKT permitirá aos profissionais de saúde delinear uma estratégia interventiva mais personalizada, dirigindo a informação transmitida às reais dificuldades do doente. ${ }^{2-5}$ Conhecendo o tipo de alimentos a evitar, reconhecendo sinais e sintomas de hipoglicemia e sabendo como agir em caso de alteração no esquema de toma normal de medicação, o diabético terá um maior controlo sobre a sua doença.

Desde a sua criação, o DKT foi já traduzido e validado para diabéticos tipo 2 em vários países como a Austrália, Malásia, entre outros, tendo-se verificado uma correlação significativa e positiva entre o conhecimento e a capacidade de controlo metabólico. ${ }^{8,9}$ Embora sem validação prévia no nosso país, o DKT foi já traduzido e aplicado em Portugal por $\mathrm{C}$. Morais et al, onde esta relação também se verificou. ${ }^{10}$

\section{Objetivos e hipóteses de estudo}

- Determinação da fiabilidade da Escala de Conhecimento da Diabetes (DKT);

- Inferência, através do número de respostas corretas obtidas no DKT, do grau de conhecimento detido pelos diabéticos quanto à sua patologia;

- Determinação da associação entre o conhecimento sobre a diabetes e variáveis sociodemográficas e específicas da doença na população em estudo.

\section{MATERIAL E MÉTODOS População e amostra}

Trata-se de uma amostra representativa, não probabilística, do tamanho da população de pessoas diagnosticados com DM2, insulinodependentes ou não, inscritas no Centro de Saúde (CS) da Covilhã. A população da amostra foi constituída sequencialmente pelos pacientes que em cada dia de trabalho sorteado como dia de estudo, foram atendidos no CS da Covilhã até ser atingido o tamanho previamente definido para um intervalo de confiança de $95 \%$ e uma margem de erro de $10 \%$, com uma perspetiva de resposta de $50 \%$, com um $n=73$, utilizando a metodologia descrita num instrumento web por Lenth. ${ }^{11}$

\section{Critérios de inclusão e exclusão}

Como critério de inclusão definiu-se: utentes adultos ( $\geq 18$ anos) com DM2 que atenderam à unidade local de saúde da Covilhã, no período compreendido entre janeiro e agosto de 2015.

Como critério de exclusão estabeleceu-se: utentes com défices cognitivos que colocassem em causa a fiabilidade do seu consentimento e a compreensão dos conceitos a avaliar.

\section{Instrumento de colheita de dados}

Para a recolha de dados foi utilizado o DKT, inquérito já validado em diversos países e agora traduzido para português (Apêndice 1, disponível em: http://www. actamedicaportuguesa.com/revista/index.php/amp/article/
view/7517/7517_Appendix01.pdf). Este documento é composto por 23 perguntas: as primeiras 14 respondidas por não insulinotratados e a totalidade das questões por insulinotratados.

Este questionário pode ser dividido em seis partes:

- Alimentação: as quatro questões iniciais baseiam-se no conhecimento sobre o tipo de alimentos a evitar, desde a sua composição à sua segurança;

- Meios de avaliação da doença: As quinta e sexta questões debruçam-se sobre os métodos utilizados para a avaliação da DM;

- Efeito de variáveis externas no controlo da patologia: Da sétima questão até à $12^{\mathrm{a}}$, o utente é questionado sobre o efeito que variáveis como o exercício e a infeção têm no controlo dos níveis de glicose;

- Sinais e sintomas: da $13^{a}$ questão à $15^{a}$ é abordado o conhecimento quanto à sintomatologia associada ao descontrolo e a evolução natural da doença;

- Controlo sobre a medicação e seus efeitos: Da questão $16^{a}$ à $21^{a}$, avalia-se a forma como o paciente atua perante adversidades e lapsos na toma da insulina;

- Causas do descontrolo glicémico: As duas últimas questões pretendem estimar a perceção quanto a possíveis etiologias da alteração do valor glicémico.

Associada à versão final ao inquérito encontrava-se uma folha a preencher pelo utente com os dados necessários para o estudo das variáveis propostas.

\section{Aplicação do instrumento de colheita de dados e verifi- cação da consistência interna}

O inquérito foi distribuído pela investigadora após o preenchimento do consentimento informado.

Especificamente para o cálculo do alfa ( $\alpha$ ) de Cronbach, que permite verificar a consistência interna do teste, este processo foi duplamente aplicado aos primeiros 18 participantes - a primeira vez de forma oral pela investigadora, e 15 minutos depois de forma escrita. Tal permitiu estimar a fiabilidade do questionário posteriormente aplicado aos restantes voluntários do estudo.

Após consentimento do voluntário, foi cedido à investigadora, pelo detentor do processo clínico, o valor de $\mathrm{HbA} 1 \mathrm{c}$, calculado como a média dos últimos dois registos analíticos.

\section{Análise estatística}

O tratamento de dados foi realizado através do recurso ao programa informático IBM SPSS Statistics - version 21.0 e Microsoft Office Excel. Aplicou-se uma estatística descritiva e inferencial, pela correlação de Pearson e, para variáveis numéricas contínuas normais, por $t$ de Student. Para verificação de consistência interna foi usado o teste a de Cronbach. Foi ainda utilizado o $\chi^{2}$. A normalidade dos dados para aplicação de inferenciação foi verificada pelo teste de Kolmogorov-Smirnov. Para uma significância estatística foi considerado o valor $p<0,05$. 


\section{Hipóteses de estudo}

Pretende-se, de acordo com a literatura científica assinalada, verificar a relação existente entre a quantidade de respostas corretas obtidas no inquérito e certas variáveis, a referir:

- $\mathrm{H} 1$ : valor de $\mathrm{HbA1c}$ - menor controlo da DM aquando de um baixo conhecimento sobre a patologia ${ }^{7,12-14}$;

- H2: sexo - melhores resultados para o sexo feminino, comparativamente ao sexo masculino ${ }^{15}$;

- H3: anos de diagnóstico de DM2 - relação positiva entre os anos de diagnóstico de DM e os conhecimentos sobre a doença ${ }^{7,15}$;

- H4: via de tratamento - melhores resultados em insulinotratados ${ }^{2,15}$

- H5: idade - relação inversa entre a idade e o conhecimento relativo à $\mathrm{DM}^{7,14,15}$;

- H6: naturalidade - piores resultados para a população diabética rural ${ }^{15}$;

- H7: existência de complicações - associação entre presença de complicações e piores desempenhos no DKT $^{7,14,15}$;

- H8: estado civil - piores resultados em pacientes viúvos. ${ }^{16,17}$

\section{RESULTADOS}

\section{Caraterização geral da amostra}

Para este estudo contribuíram 76 pessoas entre os $35 \mathrm{e}$ 87 anos, sendo a idade média dos participantes igual a 68 anos, com um desvio-padrão de 10 anos. Na caracterização da amostra, descrever apenas que $65 \%$ dos inquiridos apresentavam idade $\geq 65$ anos.

Nesta amostra populacional foi ainda possível constatar que a maioria dos utentes se enquadrava nos seguintes grupos:

Tabela 1 - Valor de alfa de Cronbach para cada pergunta do DKT, versão portuguesa

\begin{tabular}{cccc}
\hline Variável & $\begin{array}{c}\text { Valor } \\
\alpha \text { de Cronbach }\end{array}$ & Variável & $\begin{array}{c}\text { Valor } \\
\alpha \text { de Cronbach }\end{array}$ \\
\hline Q1 & 0,878 & Q13 & 0,875 \\
Q2 & 0,903 & Q14 & 0,870 \\
Q3 & 0,939 & Q15 & 0,938 \\
Q4 & 0,742 & Q16 & 0,974 \\
Q5 & 0,802 & Q17 & 0,895 \\
Q6 & 0,683 & Q18 & 0,752 \\
Q7 & 0,780 & Q19 & 0,656 \\
Q8 & 0,945 & Q20 & 0,733 \\
Q9 & 0,889 & Q21 & 0,688 \\
Q10 & 0,940 & Q22 & 0,375 \\
Q11 & 0,871 & Q23 & 0,932 \\
Q12 & 0,799 & & \\
\hline
\end{tabular}

- Sexo feminino (61\%);

- $\mathrm{HbA} 1 \mathrm{c} \leq 7 \%(57 \%)$;

- Habitante do meio rural (53\%);

- Casado(a) (73\%);

- Segundo ciclo completo como habilitação literária $(42 \%)$;

- Diagnóstico de DM2 há mais de 10 anos (50\%);

- Tratamento via oral (80\%);

- Sem complicações por DM2 (78\%).

\section{Análise de consistência interna do DKT}

Com o intuito de verificar a fidedignidade do DKT na sua versão portuguesa, recorreu-se ao $\alpha$ de Cronbach para estimar a sua consistência interna. ${ }^{18} \mathrm{~A}$ maioria das questões obteve um valor de $\alpha>0,800$, sendo o valor médio das 23 perguntas igual a 0,818, segundo a Tabela 1 .

\section{Análise dos dados face às hipóteses colocadas}

De forma a conseguir definir uma escala de resultados que permitisse uma seriação segundo o desempenho no DKT, adotamos a classificação já utilizada por Mufunda ${ }^{19}$.

Para as primeiras 14 questões do teste, consideramos um número de respostas corretas:

- < 7: conhecimento escasso;

- 7 - 11: conhecimento mediano;

-> 11: bom conhecimento.

Já no que concerne ao total das 23 questões, a organização das categorias perfilhada foi a seguinte:

- < 11: conhecimento escasso;

- 11 - 17: conhecimento mediano;

->17: bom conhecimento.

Tendo por base a definição da American Diabetes Association (ADA) e da European Association for the Study of Diabetes (EASD), consideramos 'diabético controlado' aquele que apresenta, segundo a idade, os seguintes valores de $\mathrm{HbA} 1 \mathrm{c}^{20}$ :

- Idade < 65 anos, HbA1c $\leq 7 \%$;

- Idade $\geq 65$ anos, $\mathrm{HbA} 1 \mathrm{c} \leq 8 \%$.

\section{H1: valor de HbA1c}

Para o grupo de pessoas que responderam a 14 questões, aquelas cuja DM2 se considera controlada tiveram uma média de 7,88 respostas corretas, comparativamente à média de 7,24 alcançada pelo grupo de voluntários fora dos valores de $\mathrm{HbA} 1 \mathrm{c}$ tidos como normais. Utilizando o teste $t$ de Student, reconhecemos uma relação positiva entre o conhecimento e o controlo da diabetes em não insulinotratados, embora a diferença não seja significativa.

Contudo, englobando o domínio da insulina, a relação é inversa, visto que aqueles que detêm um maior conhecimento (média de 8,65 respostas corretas) foram os que apresentaram um pior controlo sobre a sua doença. Porém, sem significância estatística.

Atendendo à correlação de Pearson ( $\rho$ de Pearson) denotamos que a interdependência existente é fracamente negativa $(\rho=-0,153)$, mas sem significância estatística. Ou seja, à medida que o valor de $\mathrm{HbA} 1 \mathrm{c}$ aumenta, o número de 

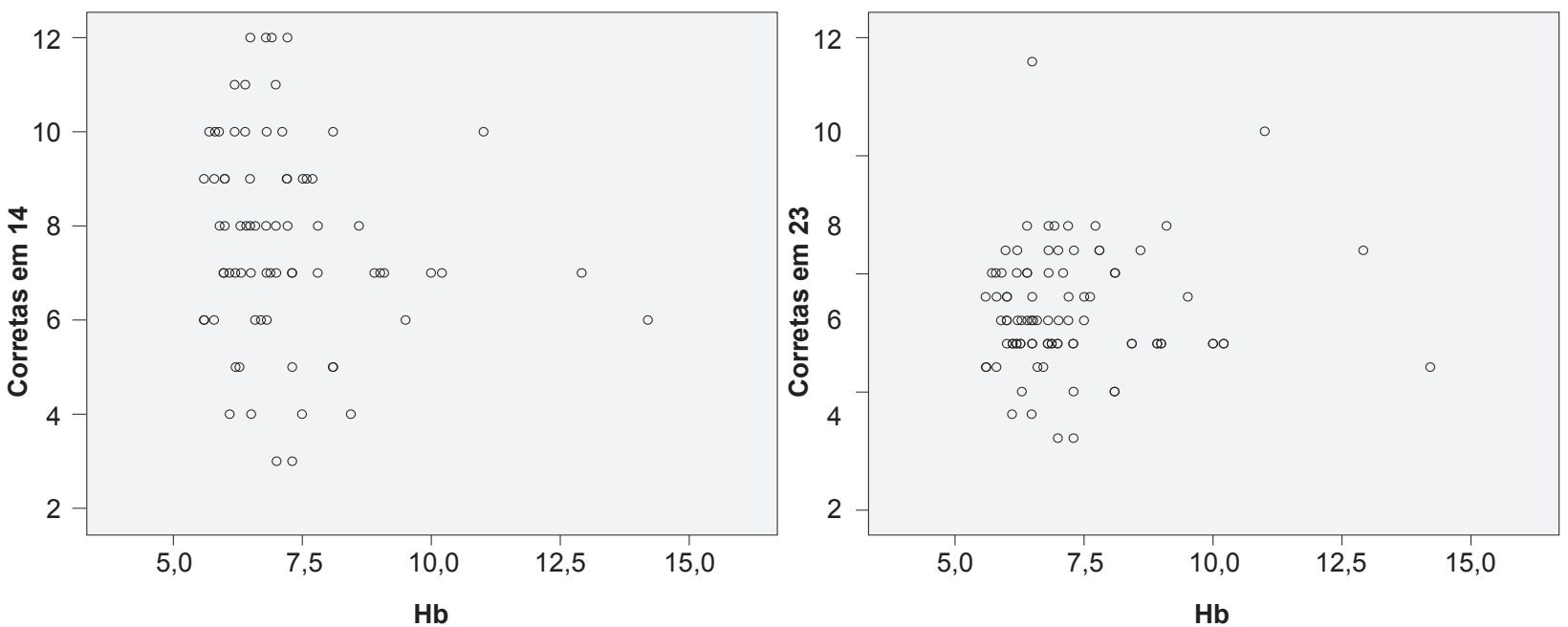

Figura 1 - Correlação de Pearson entre o valor de hemoglobina glicosilada e o número de respostas corretas em 14 e 23 questões do DKT

respostas corretas em 14 questões diminui (Fig. 1).

Já para o total das 23 questões, a $\rho$ de Pearson demonstra que a interdependência existente é medianamente positiva ( $\rho$ de Pearson $=0,083$ ), mas não significativa. Tal sugere que o aumento do valor de $\mathrm{HbA} 1 \mathrm{c}$ é acompanhado de um aumento no número de respostas corretas.

\section{H2: sexo}

Relativamente ao sexo, o sexo feminino apresentou uma média de 7,74 questões corretas face às 7,73 do sexo masculino, quando consideradas apenas as primeiras 14 questões do inquérito. Porém, este teorema altera-se quan- do os dados da amostra englobam as 23 questões. Neste grupo, o sexo masculino acerta uma média de 8,67 versus 8,48 respostas corretas do sexo feminino. Não obstante, as relações estabelecidas não apresentam significado estatístico (Fig. 2).

\section{H3: anos de diagnóstico de DM2}

Relativamente ao tempo decorrido desde o diagnóstico, os diabéticos com valores de $\mathrm{HbA} 1 \mathrm{c}$ mais controlados são aqueles cuja descoberta da patologia é mais recente $(3,19$ anos face aos 3,24 anos dos diabéticos não controlados), porém, sem significado estatístico segundo o teste ANOVA.

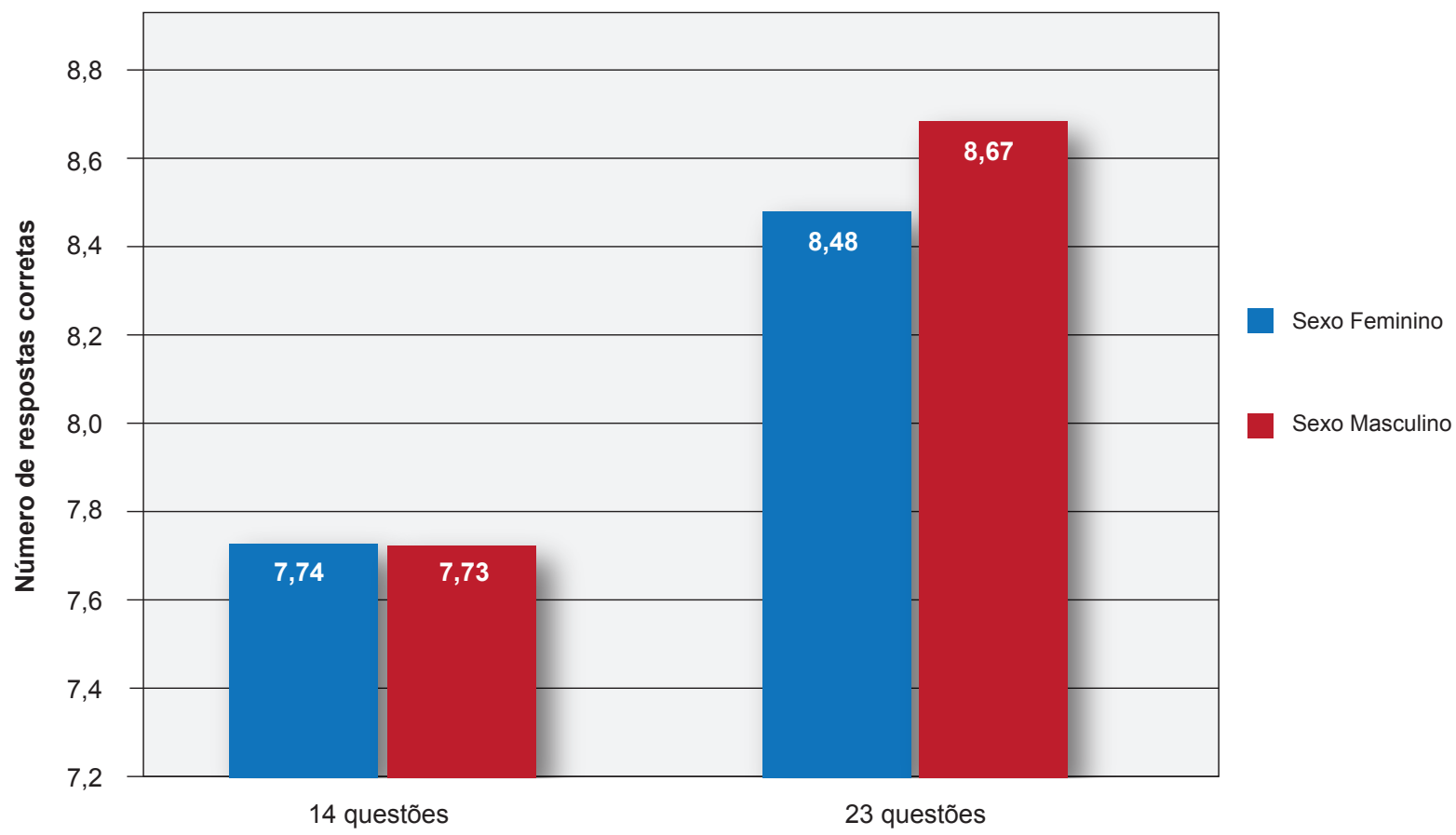

Número de respostas dadas

Figura 2 - Relação entre respostas corretas no DKT e o género 
Tabela 2 - Teste ANOVA: Relação entre respostas corretas no DKT e os anos de diagnóstico de DM2

\begin{tabular}{rcc}
\hline Anos de diagnóstico & F (ANOVA) & Valor $\boldsymbol{p}$ \\
\hline Corretas em 14 & 5,5 & 0,002 \\
Corretas em 23 & 2,1 & 0,104 \\
\hline
\end{tabular}

Considerando a Tabela 2, podemos constatar que, para a amostra que apenas respondeu a 14 questões, existe uma diferença significativa em função do tempo decorrido desde o diagnóstico.

No entanto, quanto ao conjunto das 23 questões não podemos concluir que se verifiquem diferenças com significado estatístico entre o número de respostas corretas e os vários anos de patologia diabética.

\section{H4: via de tratamento}

Para o grupo de 14 questões, a maioria dos participantes apresentou um conhecimento mediano, obtendo um total de sete a 11 respostas corretas. Neste conjunto, apenas sete atingiram o estatuto de bons conhecedores, sendo que destes, seis utilizavam um tratamento por via oral e apenas um por via injetável subcutânea.

Em relação ao grupo de 23 questões, a realidade inverte-se, dado que 59 dos voluntários demonstram um conhecimento escasso (maioria sob tratamento oral) e somente um tratado por via injetável se destacou com um número superior a 17 questões corretas.

Embora sem significância estatística no conjunto das 14 questões, o grupo do tratamento por via oral apresentou uma maior taxa de sucesso nas respostas corretas. Não obstante, no grupo de 23 questões esta relação manteve-se, sendo já estatisticamente significativa (Tabela 3 , teste ANOVA).

\section{H5: idade}

A média de idade de pacientes com a diabetes controlada foi de 68 anos, enquanto a dos não controlados foi de
Tabela 3 - Teste ANOVA: Relação entre respostas corretas no DKT e as diferentes formas de tratamento

\begin{tabular}{rcc}
\hline Formas de tratamento & F (ANOVA) & Valor $\boldsymbol{p}$ \\
\hline Corretas em 14 & 0,9 & 0,433 \\
Corretas em 23 & 12,0 & $<0,001$ \\
\hline
\end{tabular}

66 anos, ou seja os mais velhos são os que detêm a DM2 sobre um controlo mais restrito. Não obstante, esta inferência não apresenta significado estatístico, segundo o teste $t$ de Student.

Extrapolando o controlo da $\mathrm{HbA} 1 \mathrm{c}$ ao longo das diferentes faixas etárias para o conhecimento que os diabéticos tipo 2 adquirem com o passar dos anos, a média de respostas corretas não apresenta diferenças estatísticas significativas nos dois conjuntos de questões de acordo com o teste $t$ de Student.

\section{H6: naturalidade}

Observamos que os habitantes de zonas rurais foram aqueles que demonstraram ser mais cumpridores no controlo da HbA1c (82,5\% face aos $72,2 \%$ dos habitantes de zonas urbanas). Contudo, não se verificou significância estatística, segundo o teste $U$ de Mann-Whitney.

Relativamente ao conhecimento por estes detidos, observamos ser superior o desempenho dos habitantes rurais no DKT, tanto nas 14 (média de 8,30 questões corretas versus 7,11 dos urbanos) como nas 23 questões (média de 9,20 questões corretas versus 7,83 dos urbanos), com significância estatística (valores de $p$ igual a 0,015 e 0,026, respetivamente).

\section{H7: existência de complicações}

Embora a parte da amostra que apresenta retinopatia associada a pé diabético ou a nefropatia seja a que detém uma maior percentagem de pacientes controlados $(100 \%)$ - valor de $p=0,567 \cup$ no teste $U$ de Mann-Whitney, (Fig. 3) constatamos que os primeiros eram aqueles que

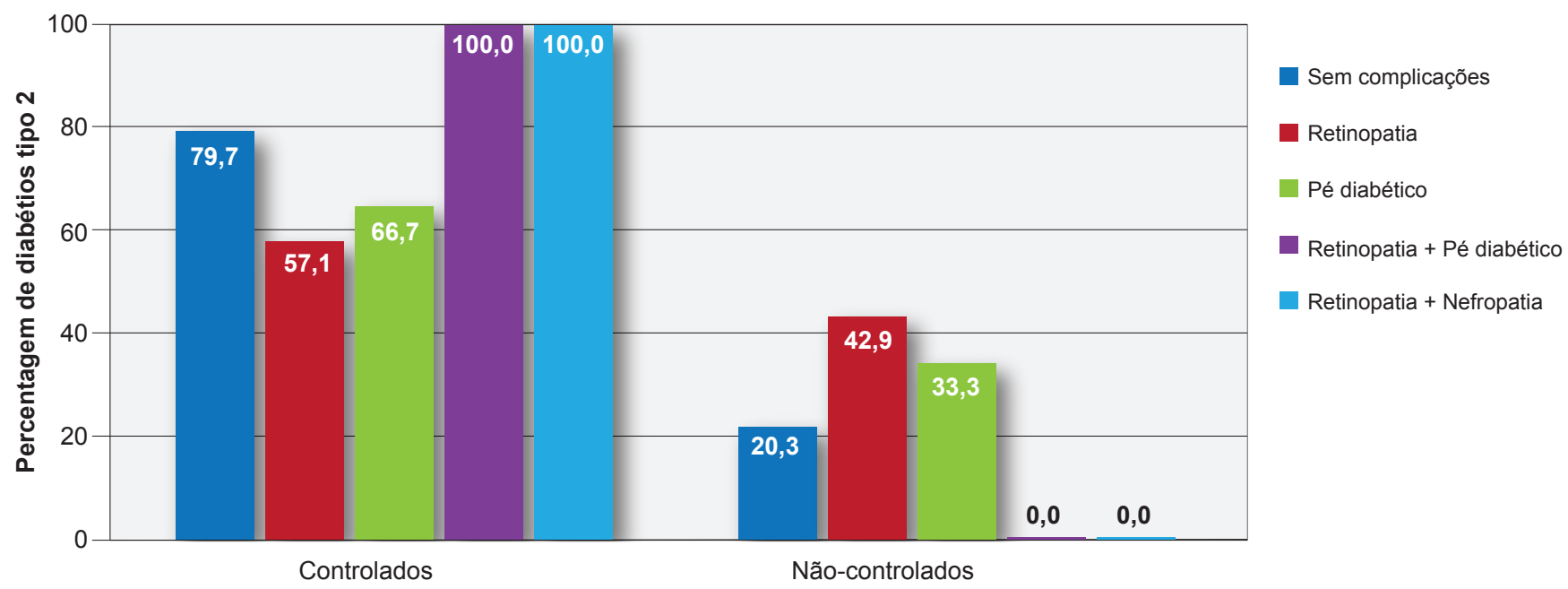

Tipo de controlo sobre a DM2

Figura 3 - Relação entre o controlo da DM2 e a presença ou não de complicações a ela secundárias 
apresentavam um pior desempenho no DKT (100\% acertava menos de 7 perguntas).

Verificamos ainda que quem não tem complicações tem um desempenho melhor no inquérito, com diferença significativa (valor $p<0.05$ no teste $t$ de Student), tanto no que respeita às 14 como às 23 questões.

\section{H8: estado civil}

Utilizando o teste de $\mathrm{U}$ de Mann-Whitney, a maioria dos inquiridos, independentemente do seu estado civil, apresenta um valor de $\mathrm{HbA} 1 \mathrm{c}$ dentro da normalidade $79 \%$ dos casados, $75 \%$ dos divorciados e $79 \%$ dos viúvos). Apenas os solteiros apresentam uma distribuição equitativa a nível do controlo metabólico ( $50 \%$ controlados e $50 \%$ fora do limite de normalidade de HbA1c), $\operatorname{com} p=0,771$.

A maioria dos participantes apresenta um nível de conhecimento moderado (50 voluntários nas primeiras 14 questões e 61 na totalidade do inquérito) sobre esta temática. Apenas sete participantes - nas primeiras 14 questões - e um - em todo o DKT - obtiveram um número suficiente de respostas certas para se denotar a sua boa compreensão sobre a DM2.

Para o conjunto de 14 questões, apenas um em 14 viúvos, face aos seis em 56 casados, obteve um total de respostas corretas $\geq 11$, pelo que os primeiros são os que demonstraram pior desempenho no inquérito. Os restantes seis voluntários, quatro divorciados e dois solteiros apresentaram um conhecimento intermédio, acertando entre 7 - 11 questões. O mesmo se verificou na totalidade do questionário, em que nenhum viúvo obteve mais de 17 respostas corretas, enquanto um casado em 56 demonstrou um bom conhecimento quanto à sua doença. Novamente, os quatro divorciados e os dois casados da amostra acertaram 11 - 17 questões, o que corresponde a um conhecimento intermédio sobre a diabetes.

\section{DISCUSSÃO}

\section{Fidedignidade da versão traduzido do DKT}

Atendendo aos valores obtidos através do cálculo do a de Cronbach para a amostra considerada, foi possível denotar que a maioria das questões obteve um valor de $\alpha>$ 0,800 o que demonstra a fidelidade psicométrica moderada a elevada do teste, confirmando assim a sua validade para o nosso país. ${ }^{18}$

\section{Relação entre os resultados do estudo e as hipóteses colocadas}

\section{H1: valor de HbA1c}

Relativamente à relação entre o valor de $\mathrm{HbA} 1 \mathrm{c}$ e o desempenho dos voluntários no DKT, verificamos que, no grupo de pessoas que responderam a 14 questões, os que obtinham um melhor resultado eram na realidade aqueles que apresentavam um melhor controlo da sua DM2. Embora estes dados não apresentem significância estatística, estão de acordo com a hipótese defendida por Fenwick et $a{ }^{\top}$ e Al-Qazaz et $a^{/ 13}$ de que a valores $\leq 7 \%-8 \%$ de $\mathrm{HbA} 1 \mathrm{c}$ (conforme a idade) se associa uma relação positiva com o conhecimento.

Porém, quando confrontados com o domínio da insulina, a relação já é inversa, sendo que a um maior conhecimento se correlaciona um pior controlo sobre a sua patologia, ainda que a diferença no número de respostas corretas face aos controlados seja mínima. Apesar de tal ser contra o acima objetivado, esta observação poderá dever-se ao facto de que apenas os insulinotratados respondiam à totalidade do inquérito, o que Ihes permitia obter um melhor resultado dado o aumento de questões a responder.

\section{H2: sexo}

Relativamente às questões gerais sobre DM2, o sexo feminino demonstrou um melhor resultado no DKT, indo ao encontro do teorema apresentado por Moodley et al. ${ }^{15} \mathrm{Po}-$ rém, o mesmo não se verificou no total das 23 questões, existindo uma ligeira superioridade do sexo masculino, o que já suporta os resultados apresentados por Tang et a/5 $\mathrm{e}$ Schillinger et al. ${ }^{14}$

Esta diferença a nível dos sexos pode estar associada ao facto de as primeiras questões explorarem mais a vertente generalista da patologia, dando relevância a temas como a alimentação e os cuidados a ter no controlo das complicações, que normalmente são mais do interesse feminino. Não obstante, as relações estabelecidas não apresentam significado estatístico, além de que não foram ainda exploradas as razões para tal discrepância.

\section{H3: anos de diagnóstico de DM2}

Como seria expectável pelas investigações de Schillinger et $a l^{14}$ e Moodley et $a l^{15}$, este estudo demonstrou que existe uma diferença significativa no desempenho dos voluntários no DKT em função do tempo decorrido desde o seu diagnóstico, na amostra que apenas respondeu a 14 questões. Desta forma, os dados sugerem que com maior tempo de evolução de doença, os conhecimentos sobre a mesma aumentam.

No entanto, quanto ao conjunto das 23 questões não existe relação entre o conhecimento e os anos de diabetes, o que vai ao encontro do trabalho de Fenwick et al. ${ }^{7}$ Segundo este, o desempenho no DKT não está associado à duração da patologia.

Porém, nesta amostra, foi possível observar que a um menor tempo de diagnóstico se associa um maior rigor na manutenção dos valores de $\mathrm{HbA} 1 \mathrm{c}$ dentro da normalidade. Embora se esperasse o contrário, dado o pressuposto de que o conhecimento adquirido ao longo dos anos de diagnóstico permitiria um melhor controlo da diabetes, tal pode dever-se ao facto de inicialmente a preocupação do diabético com a sua doença ser maior, levando a um melhor cuidado da sua saúde.

\section{H4: via de tratamento}

A maior diferença entre diabéticos controlados versus não-controlados ocorre naqueles que utilizam a via oral como única via de tratamento, não se verificando, contudo, uma diferença estatística significativa. 
Extrapolando o controlo da DM2 para o conhecimento detido pelos participantes é percetível, no grupo de 14 questões, que a maioria apresenta um conhecimento mediano, enquanto no grupo de 23 questões a realidade inverte-se, sendo predominante o conhecimento escasso. Apesar desta realidade, a via oral é a que mais acerta.

Esta conclusão vai contra a conjetura apresentada por Hess et $a{ }^{2}$ e Moodley et $a l^{15}$ que defendiam um melhor desempenho por parte dos insulinotratados, dada a necessidade de um melhor acompanhamento e maior instrução. Embora a amostra de insulinotratados seja pequena, o que pode provocar um viés a nível de resultados, esta discordância de hipóteses pode dever-se a um discurso por parte dos profissionais de saúde mais dirigido ao manuseio da insulina, descurando o ensino sobre os comportamentos modificáveis por uma possível 'descrença' no seu peso na reversão da patologia; ou ao facto de estes pressuporem o defendido pelos autores citados, subestimando o conhecimento dos pacientes.

\section{H5: idade}

Os mais velhos foram os que se destacaram pelo controlo mais rigoroso dos valores de $\mathrm{HbA} 1 \mathrm{c}$, sendo que a média de respostas corretas no DKT não apresentou diferenças estatísticas significativas para o fator idade.

Embora Fenwick et $a{ }^{1},{ }^{7}$ Moodley et $a{ }^{15}$ e Schillinger et $\mathrm{a}^{14}$ defendessem que o aumento da idade estava associado a um pior conhecimento sobre a DM2, neste estudo apenas verificamos que a idade não tinha impacto nos resultados alcançados no DKT. As possíveis explicações passam pelo facto de a capacidade de assimilação de informação pelos mais novos poder ser equilibrada por um maior enfoque educacional, por parte dos profissionais de saúde, nos grupos etários mais avançados dada a sua assumida vulnerabilidade. Assim, um provável discurso constante sobre a DM2 e suas implicações poderá estar na base de um melhor controlo e de um conhecimento equiparável aos dos mais novos por parte dos mais idosos.

\section{H6: naturalidade}

Os habitantes de zonas rurais foram aqueles que demonstram ser mais cumpridores no controlo da doença e ter um desempenho superior tanto nas 14 como nas 23 questões que compõe o DKT, com significado estatístico. Esta observação contraria o estudo de Moodley et a/15 que atribuía à população urbana um maior conhecimento sobre a patologia. Contudo, o fator responsável por esta disparidade de resultados pode assentar no pressuposto de que os residentes em zonas urbanas têm um maior grau de educação e um maior acesso à informação, sendo negligenciada a sua formação por parte dos profissionais de saúde.

\section{H7: existência de complicações}

Relativamente ao controlo da DM2, os participantes que apresentavam como complicações a retinopatia associada a pé diabético ou a nefropatia eram os que detinham os valores de HbA1c dentro dos parâmetros considerados normais. Não obstante, aqueles que mostravam retinopatia e pé diabético eram também os que exibiam um pior desempenho no DKT nos dois grupos de questões.

Em suma, o que se verificou foi que quem não tem complicações obtém um desempenho melhor no DKT, o que apoia os dados apresentados por Fenwick et al, Schillinger et al e Moodley et al ${ }^{7,14,15}$

\section{H8: estado civil}

Face à variável 'estado civil', a maioria dos participantes apresenta valores de $\mathrm{HbA} 1 \mathrm{c}$ dentro da normalidade, independentemente do seu estado civil. Contudo, a nível de conhecimento sobre a patologia, o grupo dos viúvos destaca-se ao apresentar um pior desempenho no inquérito, enquanto os restantes demonstram um nível de conhecimento moderado sobre a DM, de encontro com os resultados obtidos por Jackson et al. ${ }^{16}$

Apesar de não ter repercussões a nível metabólico, o pouco conhecimento por parte dos viúvos poderá dever-se à vulnerabilidade característica deste grupo, que pode levar a um simples cumprimento da terapêutica e das recomendações médicas gerais para a patologia, sem procurar conhecer o porquê das suas limitações. Esta interpretação dos dados é meramente especulativa, abrindo portas a futuros trabalhos.

\section{Limitações do estudo}

Mesmo tratando-se de uma amostra de tamanho representativo, o facto de esta não ser aleatória poderá determinar uma condição limitante do estudo. Além disso, tendo em consideração que a distribuição dos questionários fora feita pela investigadora, existe a probabilidade de ter ocorrido involuntariamente um potencial viés de seleção.

Outras condições limitantes são o número reduzido da amostra e a diminuta representação de insulinotratados, o que pode afetar a análise obtida e, de alguma forma, mascarar a realidade do controlo e conhecimento que os diabéticos detêm sobre a doença.

A colheita de informação relativa à presença de complicações realizou-se através do auto-reporte por parte do doente, pelo que se admite a possibilidade de um subconhecimento face à realidade que poderá ter enviesado os resultados obtidos.

\section{CONCLUSÃO}

Este estudo permitiu verificar a nível populacional a fiabilidade do DKT, criando assim mais um instrumento de avaliação clínica que possibilita aos profissionais de saúde objetivar a consciência individual dos seus utentes sobre a DM e a forma como a vivem.

A sua aplicação a uma pequena amostra de diabéticos permitiu desde já identificar grupos que precisarão de uma maior atenção por parte dos profissionais de saúde, no que respeita ao ensino, sobretudo da alimentação e do uso correto da terapêutica. De facto, ao contrário do que seria expectável, aqueles com um tempo de diagnóstico 
mais longo, insulinotratados, habitantes de zonas urbanas e viúvos foram os que obtiverem piores resultados no DKT, o que demonstra uma lacuna na prestação dos cuidados de saúde, muitas vezes por pressupostos errados quanto ao domínio da patologia por parte do doente. Isto deve alertar os profissionais para a necessidade de avaliar e reavaliar individualmente os seus diabéticos, fazendo uma formação contínua quanto às especificidades da sua doença.

A utilização deste inquérito permitirá às equipas de saúde obter uma noção mais profunda das dificuldades do seu doente sofrendo de DM, possibilitando-Ihes assim direcionar a sua intervenção contra hábitos e conhecimentos que impossibilitem uma melhoria do estado clínico.

Desta forma, espera-se ver assegurado um melhor acompanhamento e aconselhamento médico que culminarão num controlo superior por parte do doente.

Tendo em consideração a limitação a nível de tempo de consulta e da capacidade de assimilação de nova informação por parte dos utentes, este inquérito servirá como diretor do ensino, evitando uma atitude de transmissão obsoleta.

Contudo, é desejável que este trabalho sirva como base de estudos futuros e de amostras mais alargadas, que permitam fundamentar estas descobertas e criar ações a nível populacional que ajudem no combate a esta epidemia.

\section{PROTECÇÃO DE PESSOAS E ANIMAIS}

Os autores declaram que os procedimentos seguidos estavam de acordo com os regulamentos estabelecidos pelos responsáveis da Comissão de Investigação Clínica e Ética e de acordo com a Declaração de Helsínquia da Associação Médica Mundial.

\section{CONFIDENCIALIDADE DOS DADOS}

Os autores declaram ter seguido os protocolos do seu centro de trabalho acerca da publicação de dados.

\section{CONFLITOS DE INTERESSE}

Os autores declaram não terem qualquer conflito de interesse relativamente ao presente artigo.

\section{FONTES DE FINANCIAMENTO}

Os autores declaram não ter recebido subsídios ou bolsas para a elaboração do artigo.

\section{REFERÊNCIAS}

1. World Health Organization. Definition, diagnosis and classification of diabetes mellitus and its complications. Part 1: Diagnosis and classification of diabetes mellitus [Internet]. Geneva, World health Organization. 1999. [Consultado 2015 dez 07]. Disponível em: http:// apps.who.int/iris/bitstream/10665/66040/1/WHO NCD NCS 99.2.pdf

2. Hess GE, Davis WK. The validation of a diabetes patient knowledge test. Diabetes Care. 1983;6:591-6.

3. Fitzgerald JT, Funnell MM, Hess GE, Barr P, Anderson RM, Hiss RG, et al. The reliability and validity of a brief diabetes knowledge test. Diabetes Care. 1998;21:706-10.

4. Torres HC, Virginia AH, Schall VT. Validation of Diabetes Mellitus Knowledge (DKN-A) and Attitude (ATT-19) Questionnaires. Rev Saude Publica. 2005;39:906-11.

5. Tang YH, Pang SM, Chan MF, Yeung GS, Yeung VT. Health literacy, complication awareness, and diabetic control in patients with type 2 diabetes mellitus. J Adv Nurs. 2008;62:74-83.

6. Ciechanowski PS, Katon WJ, Russo JE, Walker EA. The patientprovider relationship attachment theory and adherence to treatment in diabetes. Am J Psychiatry. 2001;158:29-35.

7. Fenwick EK, Xie J, Rees G, Finger RP, Lamoureux EL. Factors associated with knowledge of diabetes in patients with type 2 diabetes using the Diabetes Knowledge Test Validated with Rasch analysis. PLoS One. 2013;8:e80593.

8. Al-Qazaz HK, Sulaiman SA, Hassali MA, Shafie AA, Sundram S. The 14-item Michigan Diabetes Knowledge Test: translation and validation study of the Malaysian version. Pract Diabetes Int. 2010;27:238-42.

9. Dunn SM, Bryson JM, Hoskins PL, Alford JB, Handelsman DJ, Turtle JR. Development of the Diabetes Knowledge (DKN) scales: forms DKNA, DKNB, and DKNC. Diabetes Care. 1984;7:36-41.

10. Morais CS, Pimenta RE, Ferreira PL, Boavida JM, Amorim JP. Assessing diabetes health literacy, knowledge and empowerment in northern Portugal. In: Rocha A, Correia MA, Costanzo S, Reis PL, editores. New contributions in information systems and technologies. Cham: Springer International Publishing; 2015. p. 63-71.
11. Lenth RV. (2006-9). Java applets for power and sample size [Computer software]. [Consultado 2015 jan 19]. Disponível em http://www.stat. uiowa.edu/ rlenth/Power.

12. Sarkar U, Fisher L, Schillinger D. Is self-efficacy associated with diabetes self-management across race / ethnicity. Diabetes Care. 2006;29:8239.

13. Al-Qazaz HK, Sulaiman SA, Hassali MA, Shafie AA, Sundram S, Al-Nuri $R$, et al. Diabetes knowledge, medication adherence and glycemic control among patients with type 2 diabetes. Int J Clin Pharm. 2011;33:1028-35.

14. Schillinger D, Grumbach K, Piette J, Wang F, Osmond D, Daher C, et al. Association of health literacy with diabetes outcomes. Prim Care. 2002;288:475-82

15. Moodley L, Rambiritch V. An assessment of the level of knowledge about diabetes mellitus among diabetic patients in a primary healthcare setting. SA Fam Pr. 2007;49:16.

16. Jackson IL, Adibe MO, Okonta MJ, Ukwe CV. Knowledge of self-care among type 2 diabetes patients in two states of Nigeria. Pharm Pract. 2014;12:1-10.

17. Cornelis MC, Chiuve SE, Glymour MM, Chang SC, Tchetgen Tchetgen EJ, Liang L, et al. Bachelors, divorcees, and widowers: does marriage protect men from type 2 diabetes? PLoS One. 2014;9:e106720.

18. Maroco J, Garcia-Marques T. Qual a fiabilidade do alfa de Cronbach? Questões antigas e soluções modernas. Laboratório Psicol. 2006;4:6590.

19. Mufunda E, Wikby K, Björn A, Hjelm K. Level and determinants of diabetes knowledge in patients with diabetes in zimbabwe: a crosssectional study. Pan Afr Med J. 2012;13:78.

20. Inzucchi SE, Bergenstal RM, Buse JB, Diamant M, Ferrannini E, Nauck $\mathrm{M}$, et al. Management of hyperglycemia in type 2 diabetes: a patientcentered approach: position statement of the American Diabetes Association (ADA) and the European Association for the Study of Diabetes (EASD). Diabetes Care. 2012;35:1364-79. 